\title{
A Novel FEM-Based Dynamic Framework For Subdivision Surfaces
}

\author{
Chhandomay Mandal* ${ }^{*} \quad$ Hong Qin $^{\dagger} \quad$ Baba C. Vemuri* \\ *Department of Computer and Information Science and Engineering \\ University of Florida \\ ${ }^{\dagger}$ Department of Computer Science \\ State University of New York at Stony Brook
}

\begin{abstract}
Subdivision surfaces have been extensively used to model smooth shapes of arbitrary topology. Recursive subdivision on an userdefined initial control mesh generates a visually pleasing smooth surface in the limit. However, users have to carefully specify the initial mesh and/or painstakingly manipulate the control vertices at different levels of subdivision hierarchy to satisfy various functional and aesthetic requirements in the limit surface. This modeling drawback results from the lack of direct manipulation tools for the limit surface. In this paper, we integrate physicsbased modeling techniques with geometric subdivision methodology and present an unified approach for arbitrary subdivision schemes. Our dynamic framework permits users to directly manipulate the limit subdivision surface via physics-based "force" tools. The key contribution of this unified approach is to formulate the smooth limit surface of any subdivision scheme as a single type of novel finite elements. The geometric and dynamic features of our subdivision-based finite elements depend on the subdivision scheme involved. We present our finite element method (FEM) and formulation for the modified butterfly and Catmull-Clark subdivision schemes, and further generalize our dynamic framework for any subdivision scheme. Our FEM-based approach significantly advances the state-of-the-art of physics-based geometric modeling because (1) our dynamic framework provides a universal physicsbased solution to any subdivision scheme beyond frequently-used and popular spline-like subdivision techniques; (2) we systematically devise a natural mechanism that allows users to intuitively deform any subdivision surface; (3) we represent the smooth limit surface of any subdivision scheme using a single type of novel subdivision-based finite elements. Our experiments demonstrate that the new unified FEM-based framework promises a greater potential of subdivision techniques for solid modeling, finite element analysis, and engineering design.
\end{abstract}

\section{INTRODUCTION}

Efficiently modeling and manipulating smooth surfaces of arbitrary topology is a grand challenge to scientists and engineers in solid modeling, computer-aided design, and interactive graphics. The recursive subdivision scheme, which produces a visually pleasing smooth surface in the limit by repeated application of a fixed set of refinement rules on an user-specified initial control mesh, is well suited for this task. Despite the prevalence of diverse subdivision schemes in the computer graphics and geometric modeling literature, it is almost impossible to manipulate the limit surface (obtained through procedure-based subdivision) in a direct and natural way. The current state-of-the-art only permits modelers to interactively obtain the desired effects on the smooth surface by kinematically manipulating the control vertices at various levels of subdivision hierarchy. In this paper, we address the challenging problem of directly manipulating the limit subdivision surface at arbitrary loca- tions/areas, and offer a novel solution to this problem by embedding purely geometric subdivision schemes in a physics-based modeling framework. Unlike the existing geometric solutions that only allow operations on control vertices, our methodology and algorithms permit users to physically modify the shape of subdivision surfaces at desired locations via application of forces. Consequently, this gives the user an intuitive and natural feeling that is uniquely produced while modeling with real clay/play-dough. Additionally, we will demonstrate that the proposed model can efficiently recover shapes from a cloud of 3D points. However, prior to the technical details of our novel scheme, we shall briefly review the previous work on subdivision surfaces.

\subsection{Background}

In [4], Chaikin first introduced the concept of subdivision to the modeling community for generating a smooth curve from an arbitrary control polygon. During the last two decades, a wide variety of subdivision schemes for modeling smooth surfaces of arbitrary topology have been derived following Chaikin's pioneering work on curve generation. The existing subdivision schemes can be broadly categorized into two distinct classes namely, (1) approximating subdivision techniques, and (2) interpolating subdivision techniques.

Among the approximating schemes, the techniques of Doo and Sabin [6] and Catmull and Clark [3] generalize the idea of obtaining uniform biquadratic and bicubic B-spline patches, respectively, from a rectangular control mesh. In [3], Catmull and Clark developed an algorithm for recursively generating a smooth surface from a polyhedral mesh of arbitrary topology. The Catmull-Clark subdivision surface, defined by an arbitrary initial mesh, can be reduced to a set of standard B-spline patches except at a finite number of degenerate points. In [12], Loop presented a similar subdivision scheme based on the generalization of quartic triangular B-splines for triangular meshes. Hoppe et al. [11] further extended Loop's work to produce piecewise smooth surfaces with selected discontinuities. Halstead et al. [10] proposed an algorithm to construct a Catmull-Clark subdivision surface that interpolates the vertices of a mesh of arbitrary topology. Peters and Reif [16] proposed a simple subdivision scheme for smoothing polyhedra. Most recently, non-uniform Doo-Sabin and Catmull-Clark surfaces that generalize non-uniform tensor-product B-spline surfaces to arbitrary topologies were introduced by Sederberg et al. [22]. All the aforementioned schemes generalize recursive subdivision schemes for generating limit surfaces with a known parameterization. Various issues involved with the use of these approximating subdivision schemes for character animation were discussed at length by DeRose et al. [5].

The most well-known interpolation-based subdivision scheme is the "butterfly" algorithm proposed by Dyn et al. [8]. Butterfly method, like other subdivision schemes, makes use of a small number of neighboring vertices for subdivision. It requires simple data structures and is rather straightforward to implement. Neverthe- 
less, it needs a topologically regular setting of the initial (control) mesh in order to obtain a smooth $C^{1}$ limit surface. Zorin et al. [25] has developed an improved interpolatory subdivision scheme (which we call the modified butterfly scheme) that retains the simplicity of the butterfly scheme and results in much smoother surfaces even from irregular initial meshes. These interpolatory subdivision schemes have extensive applications in wavelets on manifolds, multiresolution decomposition of polyhedral surfaces, and multiresolution editing.

The derivation of various mathematical properties of the smooth limit surface generated by the subdivision algorithms is rather complex. Doo and Sabin [7] first analyzed the smoothness behavior of the limit surface using the Fourier transform and an eigen-analysis of the subdivision matrix. Ball and Storry [1,2] and Reif [20] further extended Doo and Sabin's prior work on continuity properties of subdivision surfaces by deriving various necessary and sufficient conditions on smoothness for different subdivision schemes. Specific subdivision schemes were analyzed by Schweitzer [21], Habib and Warren [9], Peters and Reif [17] and Zorin [26]. Most recently, Stam [23] presented a method for exact evaluation of Catmull-Clark subdivision surfaces at arbitrary parameter values.

\subsection{Motivation}

Although recursive subdivision surfaces are extremely powerful for representing smooth geometric shapes of arbitrary topology, they constitute a purely geometric representation, and furthermore, conventional geometric modeling with subdivision surfaces may be difficult for effectively representing and deforming highly complicated objects. For example, modelers are faced with the tedium of indirect shape modification and refinement through time-consuming operations on a large number of (oftentimes irregular) control vertices when utilizing typical subdivision-based modeling techniques. Despite the advent of many modern 3D graphics interaction tools, these indirect geometric operations remain non-intuitive and laborious in general. In addition, oftentimes it may not be enough to obtain the most "fair" surface that interpolates a set of (ordered or unorganized) data points. A certain number of local features such as bulges or inflections may be strongly desired while requiring geometric objects to satisfy global smoothness criteria in solid modeling and/or interactive graphics applications. In contrast, physicsbased modeling provides a superior approach to shape modeling that can overcome most of the limitations associated with traditional geometric modeling approaches. Free-form deformable models governed by the laws of continuum mechanics are of particular relevance in this context. Dynamic models respond to externally applied forces in a very intuitive manner. The dynamic formulation marries the model geometry with time, mass, damping, and constraints via a force balance equation. Dynamic models produce smooth, natural motions which are easy to control. In addition, they facilitate interaction - especially direct manipulation of complex geometries. Furthermore, the equilibrium state of the model is characterized by a minimum of the deformation energy of the model subject to the imposed constraints. The deformation energy functionals can be formulated to satisfy local and global modeling criteria, and geometric constraints relevant to shape design can also be properly imposed. The dynamic approach subsumes all of the aforementioned modeling capabilities in a formulation which grounds everything in real-world physical behavior.

Free-form deformable models were first introduced to the modeling community by Terzopoulos et al. [24], and were improved by a number of researchers over the years. Qin and Terzopoulos [18] developed D-NURBS which are very sophisticated physics-based models suitable for representing a wide variety of free-form as well as standard analytic shapes. The D-NURBS have the advantage of interactive and direct manipulation of NURBS curves and surfaces, resulting in physically meaningful thus intuitively predictable motion and shape variation. However, a severe limitation of the existing deformable models, including D-NURBS, is that they are defined on a rectangular parametric domain. Therefore, it can be very difficult to model surfaces of arbitrary genus using these models. Subdivision schemes, in contrast, can model complex surfaces of arbitrary topology, and hence are a good candidate for developing a novel physics-based model where the modeler can directly manipulate the (complicated) limit surface in an intuitive way.

Previously we had introduced dynamic Catmull-Clark subdivision surfaces $[14,19]$ where the smooth limit surface generated by the Catmull-Clark subdivision scheme was embedded in a physicsbased modeling framework. The current research differs significantly from our prior work because the approach taken in this paper is much more general. It aims to develop a systematic and universal mechanism with which any subdivision scheme can be formulated within the physics-based framework. The critical mathematical technique we resort to is finite element analysis. We shall first formulate a dynamic representation and equation for an interpolatory subdivision scheme - the modified butterfly subdivision method - where the limit surface, unlike other generalized splinebased subdivision schemes, does not have any closed-form analytic formulation. Moreover, we shall reformulate the dynamic CatmullClark subdivision surface model using this novel methodology, and describe how to develop an unified dynamic framework for any subdivision scheme. The key contribution of this unified approach is to represent the smooth limit surface of any subdivision scheme using a single type of novel finite elements. The geometric and physical features of our subdivision-based finite elements depend only on the subdivision scheme involved. Our FEM-based approach significantly advances the state-of-the-art of physics-based geometric modeling because (1) it provides a universally physics-based solution to any subdivision schemes beyond prevalent spline-like subdivision techniques; (2) a natural mechanism that allows users to intuitively deform any subdivision surface has been systematically devised; (3) the limit surface of any subdivision schemes has been represented using a single type of novel subdivision-based finite elements; and (4) our subdivision-based finite elements are potentially of great interest to FEM communities.

\subsection{Overview}

The rest of the paper is organized as follows. A dynamic framework for the interpolatory (modified) butterfly subdivision scheme is presented in Section 2. We reformulate the dynamic framework for the approximating Catmull-Clark subdivision scheme using the proposed approach in Section 3. Section 4 presents a solution on how to develop a dynamic framework for any subdivision scheme. Experimental applications are presented in Section 5. Finally, we conclude the paper in Section 6.

\section{Dynamic Butterfly Subdivision Sur- faces}

This section discusses a dynamic framework for an interpolatory subdivision scheme namely, the (modified) butterfly subdivision technique. First, a brief overview of the (modified) butterfly subdivision scheme is presented. Next, a local geometric parameterization technique for the limit surface of the (modified) butterfly subdivision is detailed. Our parameterization method is then used to derive the new triangular finite element model for butterfly-based subdivision. Finally, the implementation details are described. Note that, we will further generalize our physics-based formulation for other interpolatory subdivision schemes in Section 4. 


\subsection{Geometry of The (Modified) Butterfly Subdivi- sion}

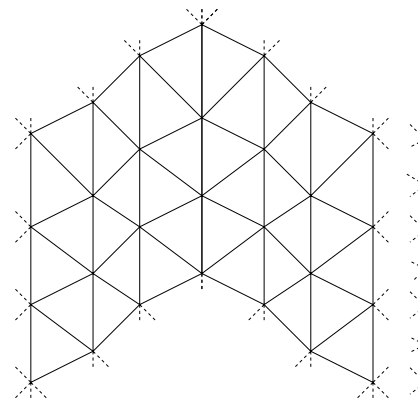

(a)

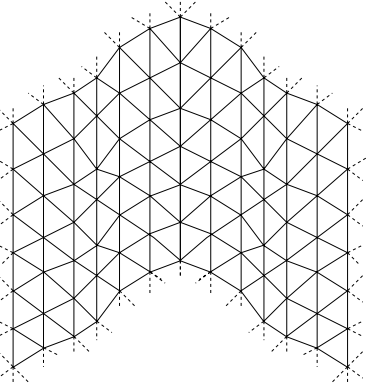

(b)
Figure 1: (a) The control polygon with triangular faces. (b) The refined mesh obtained after one subdivision step using butterfly subdivision rules.

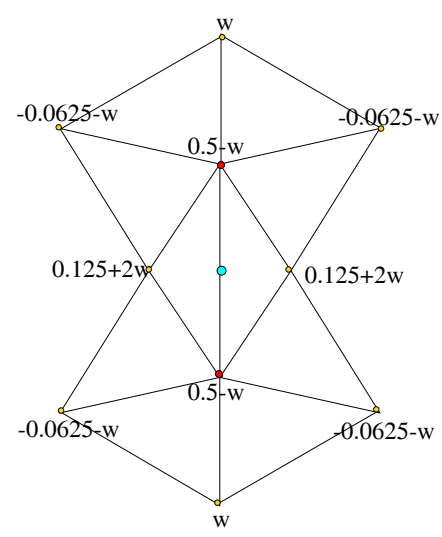

(a)

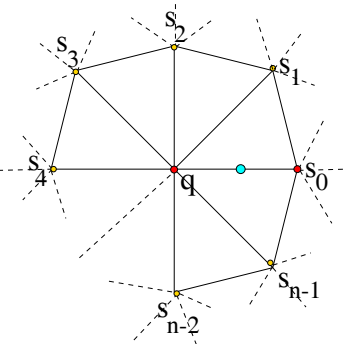

(b)
Figure 2: (a) The weighing factors of contributing vertex positions for an edge connecting two vertices of degree 6; (b) the corresponding case when one vertex is of degree $n$ and the other is of degree 6.

The butterfly subdivision scheme [8], like other subdivision techniques used in geometric modeling and graphics, starts with an initial triangular mesh (a.k.a. the control mesh) defined by a set of control vertices. In each step of subdivision, the initial (control) mesh is refined through the transformation of each triangular face into a patch with four smaller triangular faces. After one step of refinement, the new mesh in the finer level retains the vertices of each triangular face in the previous level and hence, interpolates the coarser mesh in the previous level. In addition, every edge in each triangular face is split by adding a new vertex whose position is obtained by an affine combination of the neighboring vertex positions in the coarser level. For instance, the mesh in Fig.1(b) is obtained by subdividing the initial mesh shown in Fig.1(a) once. It may be noted that all the newly introduced vertices corresponding to the edges in the original mesh have degree 6 , whereas the position and degree of all original vertices do not change in the refined mesh.

In the original butterfly scheme, the new vertices corresponding to the edges in the previous level are obtained using an eight-point stencil. It produces a smooth $C^{1}$ surface in the limit except at the extraordinary points corresponding to the extraordinary vertices (vertices with degree not equal to 6) in the initial mesh [25]. Since all the vertices introduced through subdivision have degree 6 , the number of extraordinary points in the smooth limit surface equals to the number of extraordinary vertices in the initial mesh. Recently, the original butterfly scheme has been modified by Zorin et al. [25] to obtain better smoothness properties at the extraordinary points. In this modified butterfly subdivision technique, all the edges had been categorized into three classes: (i) edges connecting two vertices of degree 6 (a 10 point stencil, as shown in Fig.2(a), is used to obtain the new vertex positions corresponding to these edges), (ii) edges connecting a vertex of degree 6 and a vertex of degree $n \neq 6$ (the corresponding stencil to obtain new vertex position is shown in Fig.2(b), where $q=.75$ is the weight associated with the vertex of degree $n \neq 6$, and $s_{i}=(0.25+\cos (2 \pi i / n)+0.5 \cos (4 \pi i / n)) / n$, $i=0,1, \ldots, n-1$, are the weights associated with the vertices of degree 6), and (iii) edges connecting two vertices of degree $n \neq 6$. The last case can not occur except in the initial mesh as the newly introduced vertices are of degree 6 , and the new vertex position in this last case is obtained by averaging the positions obtained through the use of stencil shown in Fig.2(b) at each of those two extraordinary vertices.

\subsection{Formulation}

In this section, we systematically formulate the dynamic framework for the modified butterfly subdivision scheme. Unlike the approximating schemes, the geometry of the limit surface obtained via modified butterfly subdivision does not have any closed-form analytic expression even for a regular mesh. Therefore, the key issue is to define an appropriate parametric domain and derive a local parameterization for butterfly-based subdivision. These relevant geometric components are critical to the development of our physicsbased finite element model for the limit surface of butterfly scheme.

The smooth limit surface defined by the modified butterfly subdivision technique is of arbitrary topology where a global parameterization is impossible. Nevertheless, the limit surface can be locally parameterized over the geometric domain defined by the initial mesh. The idea is to track an arbitrary point on the initial mesh across the mesh hierarchy obtained via the subdivision process (see Fig.3 and Fig.4), so that a correspondence can be established between the point being tracked in the initial mesh and its image on the limit surface.

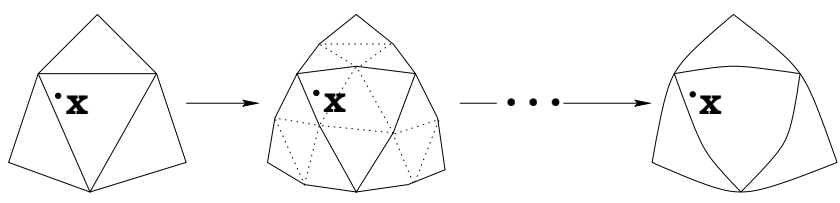

Figure 3: The smoothing effect of the subdivision process on the triangles of the initial mesh.

The modified butterfly subdivision scheme starts with an initial mesh consisting of a set of triangular faces. The recursive application of the subdivision rules smoothes out each triangular face, and in the limit, we obtain a smooth surface consists of a collection of smooth triangular patches. The subdivision process and the triangular decomposition of the limit surface is depicted in Fig.3. Note that, the limit surface can be represented by the same number of smooth triangular patches as that of the triangular faces in the initial mesh. Therefore, the limit surface $\mathbf{s}$ can be expressed as

$$
\mathbf{s}=\sum_{k=1}^{n} \mathbf{s}_{k}
$$


(a)

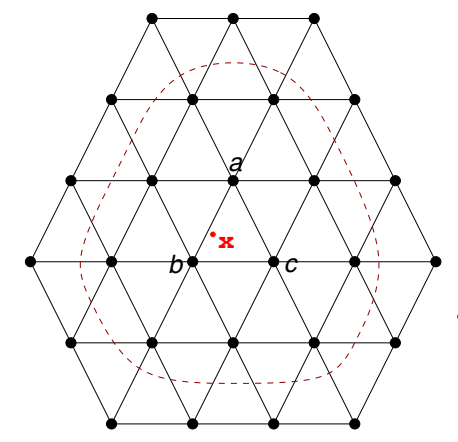

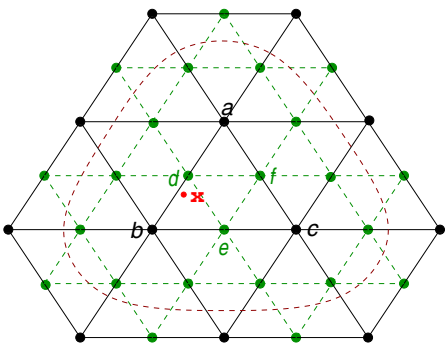

(b)

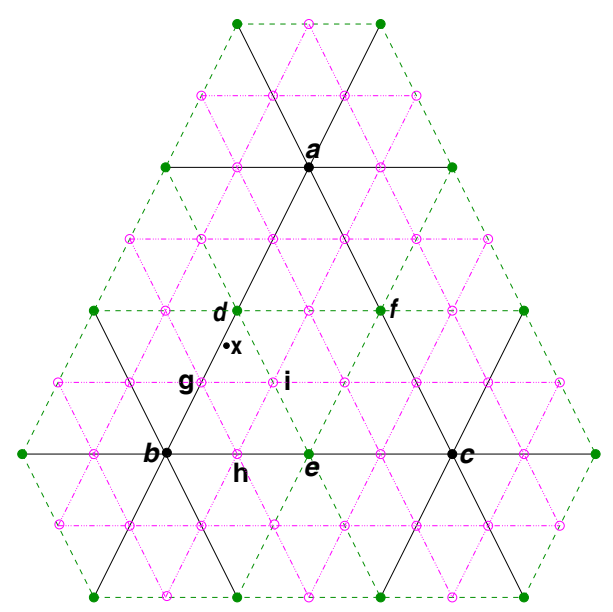

(c)

Figure 4: Tracking a point $\mathbf{x}$ through various levels of subdivision: (a) initial mesh, (b) the selected section (enclosed by dotted lines) of the mesh in (a), after one subdivision step, (c) the selected section of the mesh in (b), after another subdivision step.

where $n$ is the number of triangular faces in the initial mesh and $\mathbf{s}_{k}$ is the smooth triangular patch in the limit surface corresponding to the $k$-th triangular face in the initial mesh.

After the above geometric decomposition, we now describe the parameterization of the limit surface over the initial mesh. The procedure can be best explained through the following example. A simple planar mesh shown in Fig.4(a) is chosen as the initial mesh. An arbitrary point $\mathbf{x}$ inside the triangular face $a b c$ is tracked over the meshes obtained through subdivision. The vertices in the initial mesh are darkly shaded in Fig.4. After one step of subdivision, the initial mesh is refined by addition of new vertices which are lightly shaded. Another subdivision step on this refined mesh leads to a finer mesh with introduction of new vertices which are unshaded. It may be noted that any point inside the smooth triangular patch in the limit surface corresponding to the face abc in the initial mesh depends only on the vertices in the initial mesh which are within the 2-neighborhood of the vertices $\mathbf{a}, \mathbf{b}$ and $\mathbf{c}$ due to the local nature of the subdivision process (the $k$-neighborhood of a vertex includes all the vertices that can be reached following at most $k$ edges from the given vertex). For example, the vertex $\mathbf{d}$, introduced after first subdivision step, can be obtained using the 10 point stencil shown in Fig.2(a) on the edge $a b$. All the contributing vertices in the initial mesh are within the 1-neighborhood of the vertices a and b. A 10 point stencil can be used again in the next subdivision step on the edge $d b$ to obtain the vertex g. Some of the contributing vertices at this level of subdivision, for example, the (lightly shaded) 1-neighbors of the vertex $\mathbf{b}$ (except $\mathbf{d}$ and $\mathbf{e}$ ) in Fig.4(b), depend on some vertices in the initial mesh which are within the 2-neighborhood of the vertices $\mathbf{a}, \mathbf{b}$ and $\mathbf{c}$ in the initial mesh.

In the rest of the formulation, superscripts are used to indicate the subdivision level. For example, $\mathbf{v}_{u v w}^{j}$ denotes the collection of vertices at level $j$ which control the smooth patch in the limit surface corresponding to the triangular face $u v w$ at the $j$-th level of subdivision. Let $\mathbf{v}_{a b c}^{0}$ be the collection of vertices in the initial mesh that are within the 2-neighborhood of the vertices $\mathbf{a}, \mathbf{b}$ and $\mathbf{c}$ (marked black in Fig.4(a)). Let the number of such vertices be $r$. Then, the vector $\mathbf{v}_{a b c}^{0}$, which is the concatenation of the $(x, y, z)$ positions for all the $r$ vertices, is of dimension $3 r$. Based on the above observation of the 2-neighborhood property, the geometry of the smooth triangular patch in the limit surface corresponding to the triangular face $a b c$ in the initial mesh is uniquely determined by these $r$ vertices. Because of the recursive characteristic, there now exists four subdivision matrices $\left(\mathbf{A}_{a b c}\right)_{t},\left(\mathbf{A}_{a b c}\right)_{l},\left(\mathbf{A}_{a b c}\right)_{r}$ and $\left(\mathbf{A}_{a b c}\right)_{m}$ of dimension $(3 r, 3 r)$ such that

$$
\begin{aligned}
\mathbf{v}_{a d f}^{1} & =\left(\mathbf{A}_{a b c}\right)_{t} \mathbf{v}_{a b c}^{0} \\
\mathbf{v}_{b e d}^{1} & =\left(\mathbf{A}_{a b c}\right)_{l} \mathbf{v}_{a b c}^{0}, \\
\mathbf{v}_{c f e}^{1} & =\left(\mathbf{A}_{a b c}\right)_{r} \mathbf{v}_{a b c}^{0} \\
\mathbf{v}_{d e f}^{1} & =\left(\mathbf{A}_{a b c}\right)_{m} \mathbf{v}_{a b c}^{0}
\end{aligned}
$$

where the subscripts $t, l, r$ and $m$ denote top, left, right and middle triangle positions, respectively (indicating the relative position of the new triangle with respect to the original triangle), and $\mathbf{v}_{a d f}^{1}, \mathbf{v}_{b e d}^{1}, \mathbf{v}_{c f e}^{1}$ and $\mathbf{v}_{\text {def }}^{1}$ are the concatenation of the $(x, y, z)$ positions for the vertices in the 2-neighborhood of the corresponding triangle within the newly obtained refined mesh after one subdivision. Note that, the new vertices in this level of subdivision are lightly shaded in Fig.4(b). The 2-neighborhood configuration of the vertices in the newly obtained triangles is exactly the same as that of the original triangle, hence local subdivision matrices are square and the vector dimensions on both sides of Eqn. 2 are the same.

Carrying out one more level of subdivision, a new set of vertices which are unshaded in Fig.4(c) are obtained along with the old vertices. Adopting a similar approach as in the derivation of Eqn.2, it can be shown that

$$
\begin{aligned}
\mathbf{v}_{d g i}^{2} & =\left(\mathbf{A}_{b e d}\right)_{t} \mathbf{v}_{b e d}^{1} \\
\mathbf{v}_{b h g}^{2} & =\left(\mathbf{A}_{b e d}\right)_{l} \mathbf{v}_{b e d}^{1} \\
\mathbf{v}_{e i h}^{2} & =\left(\mathbf{A}_{b e d}\right)_{r} \mathbf{v}_{b e d}^{1} \\
\mathbf{v}_{g h i}^{2} & =\left(\mathbf{A}_{b e d}\right)_{m} \mathbf{v}_{b e d}^{1}
\end{aligned}
$$

The relative position and geometric structure of the triangular face $d g i$ in Fig.4(c) with respect to the triangular face bed is topologically the same as of the triangular face $a d f$ in Fig.4(b) with respect to the triangular face $a b c$. Therefore, we can obtain $\left(\mathbf{A}_{b e d}\right)_{t}=\left(\mathbf{A}_{a b c}\right)_{t}$. Based on the similar reasoning, Eqn.3 can be rewritten as

$$
\begin{aligned}
\mathbf{v}_{d g i}^{2} & =\left(\mathbf{A}_{b e d}\right)_{t} \mathbf{v}_{b e d}^{1}=\left(\mathbf{A}_{a b c}\right)_{t} \mathbf{v}_{b e d}^{1} \\
\mathbf{v}_{b h g}^{2} & =\left(\mathbf{A}_{b e d}\right)_{l} \mathbf{v}_{b e d}^{1}=\left(\mathbf{A}_{a b c}\right)_{l} \mathbf{v}_{b e d}^{1} \\
\mathbf{v}_{e i h}^{2} & =\left(\mathbf{A}_{b e d}\right)_{r} \mathbf{v}_{b e d}^{1}=\left(\mathbf{A}_{a b c}\right)_{r} \mathbf{v}_{b e d}^{1} \\
\mathbf{v}_{g h i}^{2} & =\left(\mathbf{A}_{b e d}\right)_{m} \mathbf{v}_{b e d}^{1}=\left(\mathbf{A}_{a b c}\right)_{m} \mathbf{v}_{b e d}^{1} .
\end{aligned}
$$

Combining Eqn. 2 and Eqn.4, it can be shown that

$$
\mathbf{v}_{d g i}^{2}=\left(\mathbf{A}_{a b c}\right)_{t}\left(\mathbf{A}_{a b c}\right)_{l} \mathbf{v}_{a b c}^{0}
$$




$$
\begin{aligned}
\mathbf{v}_{b h g}^{2} & =\left(\mathbf{A}_{a b c}\right)_{l}\left(\mathbf{A}_{a b c}\right)_{l} \mathbf{v}_{a b c}^{0} \\
\mathbf{v}_{e i h}^{2} & =\left(\mathbf{A}_{a b c}\right)_{r}\left(\mathbf{A}_{a b c}\right)_{l} \mathbf{v}_{a b c}^{0} \\
\mathbf{v}_{g h i}^{2} & =\left(\mathbf{A}_{a b c}\right)_{m}\left(\mathbf{A}_{a b c}\right)_{l} \mathbf{v}_{a b c}^{0}
\end{aligned}
$$

Let $\mathbf{x}$ be a point with barycentric coordinates $\left(\alpha_{a b c}^{0}, \beta_{a b c}^{0}, \gamma_{a b c}^{0}\right)$ inside the triangular face $a b c$. When the initial mesh is refined, $\mathbf{x}$ becomes a point inside the triangular face bed with barycentric coordinates $\left(\alpha_{b e d}^{1}, \beta_{b e d}^{1}, \gamma_{b e d}^{1}\right)$. Another level of subdivision causes $\mathbf{x}$ to be included in the triangular face $d g i$ with barycentric coordinates $\left(\alpha_{d g i}^{2}, \beta_{d g i}^{2}, \gamma_{d g i}^{2}\right)$. Let $\mathbf{s}_{a b c}^{j}$ denote the $\mathrm{j}$-th level approximation of the smooth triangular patch $\mathbf{s}_{a b c}$ in the limit surface corresponding to the triangular face $a b c$ in the initial mesh. Now $\mathbf{v}_{a b c}^{0}$ can be written as

$$
\mathbf{v}_{a b c}^{0}=[\overbrace{a_{x}, b_{x}, c_{x}, \ldots,}^{r}, \overbrace{a_{y}, b_{y}, c_{y}, \ldots,}^{r} \overbrace{a_{z}, b_{z}, c_{z}, \ldots .}^{r}]^{T}
$$

where the subscripts $x, y$ and $z$ indicate the $\mathrm{x}, \mathrm{y}$ and $\mathrm{z}$ coordinates of the corresponding vertex position,respectively. The expressions for $\mathbf{v}_{b e d}^{1}$ and $\mathbf{v}_{d g i}^{2}$ can also be written in a similar manner. Next, the matrix $\mathbf{B}_{a b c}^{0}$ can be constructed as follows:

$\mathbf{B}_{a b c}^{0}(\mathbf{x})=\left[\begin{array}{c}\overbrace{\overbrace{\alpha_{a b c}^{0}, \beta_{a b c}^{0}, \gamma_{a b c}^{0}, 0, \ldots, 0}^{r}, \overbrace{0, \ldots, 0}^{r}, \overbrace{0, \ldots, 0}^{r}}^{r}, \overbrace{\alpha_{a b c}^{0}, \beta_{a b c}^{0}, \gamma_{a b c}^{0}, 0, \ldots, 0,0, \overbrace{0, \ldots, 0}^{r}}^{r} \\ \overbrace{0, \ldots, 0}^{r}, \overbrace{0, \ldots, 0}^{r}, \overbrace{\alpha_{a b c}^{0}, \beta_{a b c}^{0}, \gamma_{a b c}^{0}, 0, \ldots, 0}^{r}\end{array}\right]$.

The matrices $\mathbf{B}_{b e d}^{1}$ and $\mathbf{B}_{d g i}^{2}$ can also be constructed in a similar fashion. Now $\mathbf{s}_{a b c}^{0}(\mathbf{x}), \mathbf{s}_{a b c}^{1}(\mathbf{x})$, and $\mathbf{s}_{a b c}^{2}(\mathbf{x})$ can be written as

$$
\begin{aligned}
\mathbf{s}_{a b c}^{0}(\mathbf{x}) & =\mathbf{B}_{a b c}^{0}(\mathbf{x}) \mathbf{v}_{a b c}^{0}, \\
\mathbf{s}_{a b c}^{1}(\mathbf{x}) & =\mathbf{B}_{b e d}^{1}(\mathbf{x}) \mathbf{v}_{b e d}^{1}=\mathbf{B}_{b e d}^{1}(\mathbf{x})\left(\mathbf{A}_{a b c}\right)_{l} \mathbf{v}_{a b c}^{0} \\
\mathbf{s}_{a b c}^{2}(\mathbf{x}) & =\mathbf{B}_{d g i}^{2}(\mathbf{x}) \mathbf{v}_{d g i}^{2}=\mathbf{B}_{d g i}^{2}(\mathbf{x})\left(\mathbf{A}_{a b c}\right)_{t} \mathbf{v}_{b e d}^{1} \\
& =\mathbf{B}_{d g i}^{2}(\mathbf{x})\left(\mathbf{A}_{a b c}\right)_{t}\left(\mathbf{A}_{a b c}\right)_{l} \mathbf{v}_{a b c}^{0}
\end{aligned}
$$

Proceeding in a similar way, the expression for $\mathbf{s}_{a b c}^{j}(\mathbf{x}), j$-th level approximation of $\mathbf{s}_{a b c}(\mathbf{x})$, is given by

$$
\begin{aligned}
\mathbf{s}_{a b c}^{j}(\mathbf{x}) & =\mathbf{B}_{u v w}^{j}(\mathbf{x}) \overbrace{\left(\mathbf{A}_{a b c}\right)_{m} \ldots\left(\mathbf{A}_{a b c}\right)_{t}\left(\mathbf{A}_{a b c}\right)_{l}}^{j} \mathbf{v}_{a b c}^{0} \\
& =\mathbf{B}_{u v w}^{j}(\mathbf{x})\left(\mathbf{A}_{a b c}^{j}\right) \mathbf{v}_{a b c}^{0} \\
& =\mathbf{B}_{a b c}^{j}(\mathbf{x}) \mathbf{v}_{a b c}^{0},
\end{aligned}
$$

where $\mathbf{x}$ is inside the triangular face $u v w$ at level $j$ (with an assumption that $u v w$ is the triangular face in the middle with respect to its coarser-level original triangular face in the previous level), $\left(\mathbf{A}_{a b c}^{j}\right)$ $=\left(\mathbf{A}_{a b c}\right)_{m} \ldots\left(\mathbf{A}_{a b c}\right)_{t}\left(\mathbf{A}_{a b c}\right)_{l}$ and $\mathbf{B}_{a b c}^{j}(\mathbf{x})=\mathbf{B}_{u v w}^{j}(\mathbf{x})\left(\mathbf{A}_{a b c}^{j}\right)$. It may be noted that the sequence of applying $\left(\mathbf{A}_{a b c}\right)_{t},\left(\mathbf{A}_{a b c}\right)_{l}$, $\left(\mathbf{A}_{a b c}\right)_{r}$ and $\left(\mathbf{A}_{a b c}\right)_{m}$ depends on the triangle inside which the tracked point $\mathbf{x}$ falls after each subdivision step. Finally, the local geometric parameterization procedure can be completed by writing

$$
\mathbf{s}_{a b c}(\mathbf{x})=\left(\lim _{j \rightarrow \infty} \mathbf{B}_{a b c}^{j}(\mathbf{x})\right) \mathbf{v}_{a b c}^{0}=\mathbf{B}_{a b c}(\mathbf{x}) \mathbf{v}_{a b c}^{0} .
$$

Note that, $\mathbf{B}_{a b c}$ is the collection of basis functions at the vertices of $\mathbf{v}_{a b c}^{0}$. It may also be noted that the modified butterfly subdivision scheme is a stationary subdivision process, and hence new vertex positions are obtained by affine combinations of nearby vertices. This guarantees that each row of the matrices
$\left(\mathbf{A}_{a b c}\right)_{t},\left(\mathbf{A}_{a b c}\right)_{l},\left(\mathbf{A}_{a b c}\right)_{r}$ and $\left(\mathbf{A}_{a b c}\right)_{m}$ sums to one. The largest eigenvalue of such matrices is 1 and therefore the mathematical limit in Eqn. 8 exists. Now, assuming the triangular face $a b c$ is the $k$-th face in the initial mesh, Eqn. 8 can be rewritten as

$$
\mathbf{s}_{k}(\mathbf{x})=\mathbf{B}_{k}(\mathbf{x}) \mathbf{v}_{k}^{0}=\mathbf{B}_{k}(\mathbf{x}) \mathbf{A}_{k} \mathbf{p},
$$

where $\mathbf{p}$ is the concatenation of the $(\mathrm{x}, \mathrm{y}, \mathrm{z})$ positions of all the vertices in the initial mesh and the matrix $\mathbf{A}_{k}$, when post-multiplied by $\mathbf{p}$, only selects the vertices $\mathbf{v}_{k}^{0}$ defining the $\mathrm{k}$-th smooth triangular patch in the limit surface. If there are $t$ vertices in the initial mesh and $r$ of them control the $k$-th patch, then $\mathbf{p}$ is a vector of dimension $3 t, \mathbf{A}_{k}$ is a matrix of dimension $(3 r, 3 t)$, and $\mathbf{B}_{k}(\mathbf{x})$ is a matrix of dimension $(3,3 r)$.

Combining Eqn.1 and Eqn.9, it can be shown that

$$
\mathbf{s}(\mathbf{x})=\left(\sum_{k=1}^{n} \mathbf{B}_{k}(\mathbf{x}) \mathbf{A}_{k}\right) \mathbf{p}=\mathbf{J}(\mathbf{x}) \mathbf{p}
$$

where $\mathbf{J}$, a matrix of dimension $(3,3 t)$, is the collection of basis functions for the corresponding vertices in the initial mesh. The vector $\mathbf{p}$ is also known as the degrees of freedom vector of the smooth limit surface $\mathbf{s}$.

We now treat the vertex positions in the initial mesh defining the smooth limit surface $\mathbf{s}$ as time variables in order to develop the new dynamic butterfly subdivision model. The velocity of the surface model can be expressed as $\dot{\mathbf{s}}(\mathbf{x}, \mathbf{p})=\mathbf{J}(\mathbf{x}) \dot{\mathbf{p}}$, where an overstruck dot denotes a time derivative and $\mathbf{x} \in S^{0}, S^{0}$ being the domain defined by the initial mesh. Note that, $S^{0}$ is the parametric domain of the limit surface, each triangle of the initial control mesh serves as a local parametric domain for its corresponding triangular patch.

\subsection{Finite Element Procedure}

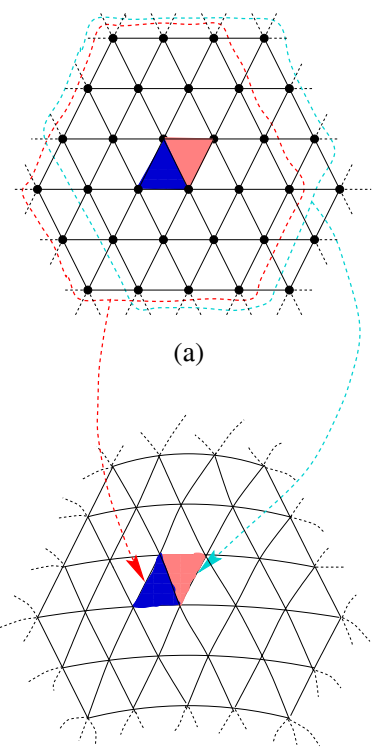

(b)

Figure 5: (a) An initial mesh, and (b) the corresponding limit surface. The domains of the shaded elements in the limit surface are the corresponding triangular faces in the initial mesh. The encircled vertices in (a) are the degrees of freedom for the corresponding element.

In Section 2.2 we have demonstrated that the smooth limit surface of butterfly subdivision can be represented by a collection of 
smooth triangular patches. In our dynamic framework, we now consider each patch of the limit surface as a finite element. The number of such patches is equal to the number of triangular faces in the initial mesh as mentioned earlier. The concept of decomposing the smooth limit surface into a collection of elements is illustrated in Fig.5. We also show the parametric domain and control vertices for shaded elements in Fig.5. The governing motion equation of this subdivision-based FEM model is given by

$$
\mathbf{M} \ddot{\mathbf{p}}+\mathbf{D} \dot{\mathbf{p}}+\mathbf{K} \mathbf{p}=\mathbf{f}_{p},
$$

where $\mathbf{f}_{p}$ is the generalized force vector, and $\mathbf{M}, \mathbf{D}$, and $\mathbf{K}$ are the mass, damping and stiffness matrices of the physical model. We provide an outline on how to derive the mass, damping and stiffness matrices for these finite elements so that a numerical solution to the governing second-order differential equation can be obtained using popular finite element analysis techniques. We use the same example as in Section 2.2 (refer to Fig.4) to introduce the relevant concepts and derive our FEM model.

The mass matrix for the element $\mathbf{s}_{a b c}$, corresponding to the triangular face $a b c$, can be expressed as

$$
\mathbf{M}_{a b c}=\int_{\mathbf{x} \in \mathbf{s}_{a b c}} \mu(\mathbf{x}) \mathbf{B}_{a b c}^{T}(\mathbf{x}) \mathbf{B}_{a b c}(\mathbf{x}) d \mathbf{x} .
$$

However, the basis functions (stored as entries in $\mathbf{B}_{a b c}$ ) do not have any analytic form, hence computing this integral is a difficult proposition. We solve this problem by approximating the smooth triangular patch in the limit surface corresponding to the face $a b c$ in the initial mesh by a triangular mesh with $4^{j}$ faces obtained after $j$ levels of subdivision of the original triangular face $a b c$ (each subdivision step splits one triangular face into 4 triangular faces). In addition, we choose a discretized form of mass distribution function which has non-zero values only at the vertex positions of the $j$-th subdivision level mesh. Then the mass matrix can be approximated as

$$
\mathbf{M}_{a b c}=\sum_{i=1}^{k} \mu\left(\mathbf{v}_{i}^{j}\right)\left\{\mathbf{B}_{a b c}^{j}\left(\mathbf{v}_{i}^{j}\right)\right\}^{T}\left\{\mathbf{B}_{a b c}^{j}\left(\mathbf{v}_{i}^{j}\right)\right\}
$$

where $k$ is the number of vertices in the triangular mesh with $4^{j}$ faces. This approximation has been found to be very effective and efficient for the implementation of FEM procedure. The computation of elemental damping matrix follows suit.

Physics-based models have both kinetic and potential energies. We now define the internal (e.g., elastic) energy of the subdivisionbased dynamic model by assigning deformation energy to each element. We take a similar approach as shown above and consider the $j$-th level approximation of the element. Note that, a wide range of functional formulations can be employed to describe various material and physical behaviors such as linear elastic deformation and non-linear plastic deformation. Throughout this paper, in particular, we assign spring-like energy to the approximated model because of its simplicity and efficient computation. The energy at the $j$-th level of approximation can be defined as

$$
\begin{aligned}
E_{a b c} \approx E_{a b c}^{j} & =\frac{1}{2} \sum_{\Omega} \frac{k_{l m}\left(\left|\mathbf{v}_{l}^{j}-\mathbf{v}_{m}^{j}\right|-\ell_{l m}\right)}{\left|\mathbf{v}_{l}^{j}-\mathbf{v}_{m}^{j}\right|}\left(\mathbf{v}_{l}^{j}-\mathbf{v}_{m}^{j}\right) \\
& =\frac{1}{2}\left\{\mathbf{v}_{a b c}^{j}\right\}^{T}\left(\mathbf{K}_{a b c}^{j}\right)\left\{\mathbf{v}_{a b c}^{j}\right\},
\end{aligned}
$$

where $k_{l m}$ is the spring-controlling variable, $\mathbf{v}_{l}^{j}$ and $\mathbf{v}_{m}^{j}$, the $l$-th and $m$-th vertex in the $j$-th level mesh, are in the 1-neighborhood of each other, $\Omega$ is the domain defined by all such vertex pairs, $\ell_{l m}$ is the natural length of the spring connected between $\mathbf{v}_{l}^{j}$ and $\mathbf{v}_{m}^{j}$, and $\mathbf{v}_{a b c}^{j}$ is the concatenation of the $(\mathrm{x}, \mathrm{y}, \mathrm{z})$ positions of all the vertices in the $j$-th subdivision level of the triangular face $a b c$ in the

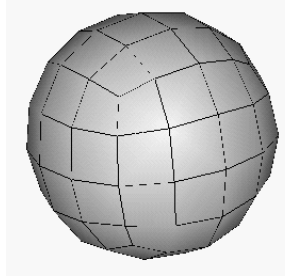

(a)

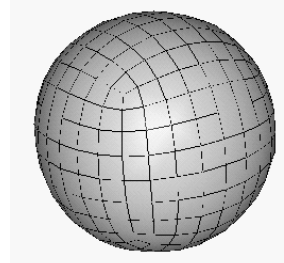

(b)

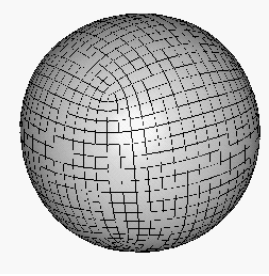

(c)
Figure 6: Catmull-Clark subdivision: (a) initial mesh, (b) mesh obtained after one step of Catmull-Clark subdivision, and (c) mesh obtained after another subdivision step.

initial mesh. The vertex positions in $\mathbf{v}_{a b c}^{j}$ are obtained by a linear combination of the vertex positions in $\mathbf{v}_{a b c}^{0}$, and hence we can write $\mathbf{v}_{a b c}^{j}=\left(\mathbf{A}_{a b c}^{j}\right) \mathbf{v}_{a b c}^{0}$ where $\left(\mathbf{A}_{a b c}^{j}\right)$ is the transformation (subdivision) matrix. Therefore, the expression for the elemental stiffness matrix is given by $\mathbf{K}_{a b c}=\left(\mathbf{A}_{a b c}^{j}\right)^{T}\left(\mathbf{K}_{a b c}^{j}\right)\left(\mathbf{A}_{a b c}^{j}\right)$. It may be noted that this approach is applicable for modeling isotropic as well as anisotropic phenomena because $k_{l m}$, the spring-controlling variable, can be a time-dependent function in general.

\section{Dynamic Catmull-Clark Subdivision Surfaces}

In this section, we consider a new FEM model based on an approximating subdivision scheme, namely, Catmull-Clark subdivision technique. Please note, the dynamic formulation of CatmullClark subdivision previously proposed in $[14,19]$ could not be generalized for other approximating subdivision schemes. The framework developed in this section can be generalized to other approximating subdivision schemes as shown in Section 4. In fact, a dynamic framework for Loop's technique (another popular approximating subdivision scheme) has been presented in [13] using the algorithm proposed here. We limit our discussion to Catmull-Clark subdivision surfaces only in this paper due to space restrictions. We first outline the Catmull-Clark subdivision scheme. Next, we present the dynamic formulation. In particular, we address the difference between the current work and prior results $[14,19]$. Finally, we discuss the finite element implementation of the physics-based model.

\subsection{Catmull-Clark Subdivision Scheme}

Catmull-Clark subdivision scheme, like any other subdivision scheme, starts with an user-defined mesh of arbitrary topology. It refines the initial mesh by adding new vertices, edges and faces with each step of subdivision following a fixed set of subdivision rules. In the limit, a sequence of recursively refined polyhedral meshes will converge to a smooth surface. The subdivision rules are as follows:

(1) For each face, a new face point is introduced which is the average of all the old vertices defining the face.

(2) For each (non-boundary) edge, a new edge point is introduced which is the average of the following four points: two old vertices defining the edge and two new face points of the faces adjacent to the edge.

(3) For each (non-boundary) vertex $V$, new vertex is introduced whose position is $\frac{F}{n}+\frac{2 E}{n}+\frac{(n-3) V}{n}$, where $F$ is the average of the 
new face vertices of all faces adjacent to the old vertex $V, E$ is the average of the midpoints of all edges incident on the old vertex $V$ and $n$ is the number of the edges incident on the vertex.

(4) New edges are formed by connecting each new face point to the new edge points of the edges defining the old face and by connecting each new vertex point to the new edge points of all old edges incident on the old vertex point.

(5) New faces are defined as faces enclosed by new edges.

An example of Catmull-Clark subdivision on an initial mesh is shown in Fig.6. The most important property of the Catmull-Clark subdivision surfaces is that a smooth surface can be generated from any control mesh of arbitrary topology. Catmull-Clark subdivision surfaces include standard bicubic B-spline surfaces as their special case (i.e., the limit surface is a bicubic B-spline surface for a rectangular mesh with all non-boundary vertices of degree 4). In addition, the aforementioned subdivision rules generalize the recursive bicubic B-spline patch subdivision algorithm. For non-rectangular meshes, the limit surface converges to a bicubic B-spline surface except at a finite number of extraordinary points. These extraordinary points correspond to extraordinary vertices (vertices whose degree is not equal to 4) in the mesh. Note that, after the first subdivision, all faces are quadrilaterals, hence all new vertices created subsequently will have four incident edges. The number of extraordinary points on the limit surface is a constant, and is equal to the number of extraordinary vertices in the refined mesh obtained after applying one step of the Catmull-Clark subdivision on the initial mesh. The limit surface is curvature-continuous everywhere except at extraordinary vertices, where only tangent plane continuity is achieved.

\subsection{Formulation}

A systematic formulation of the newly proposed dynamic framework for Catmull-Clark subdivision surfaces is presented in this section. The key difference between the dynamic model developed in $[14,19]$ and the one presented here is the representation of the limit surface. The previously proposed approach leads to diverse types of finite elements, whereas the present approach leads to single type of finite elements. This is illustrated with a schematic diagram in Fig.7.

Following the concepts developed in $[14,19]$, the limit surface of the control mesh shown in Fig.7, consists of quadrilateral bicubic B-spline patches corresponding to the faces marked 'n' (faces with no extraordinary points), and a pentagonal patch corresponding to the faces marked 's' (faces having one extraordinary vertex of degree 5) (Fig.7(a)). However, in this section, it has been shown that the entire limit surface can be expressed as a collection of quadrilateral patches as shown in Fig.7(b) using the algorithm proposed in [23]. We next discuss a local parameterization of the limit surface which is critical to embed the limit surface in a dynamic framework.

As mentioned earlier, the control mesh (after at most one subdivision step) for the Catmull-Clark subdivision scheme consists of quadrilateral faces which lead to quadrilateral patches in the limit surface. For the sake of formulation simplicity, it has been assumed that each face has at most one extraordinary vertex. If this assumption is not valid, then one more subdivision step needs to be performed on the current control mesh in order to obtain a new control mesh on which the following analysis can be carried out. The number of quadrilateral patches in the limit surface is equal to the number of non-boundary quadrilateral faces in the control mesh (Fig.8). Therefore, the smooth limit surface $\mathbf{s}$ can be expressed as

$$
\mathbf{s}=\sum_{l=1}^{n} \mathbf{s}_{l},
$$

where $n$ is the number of non-boundary faces in the control mesh

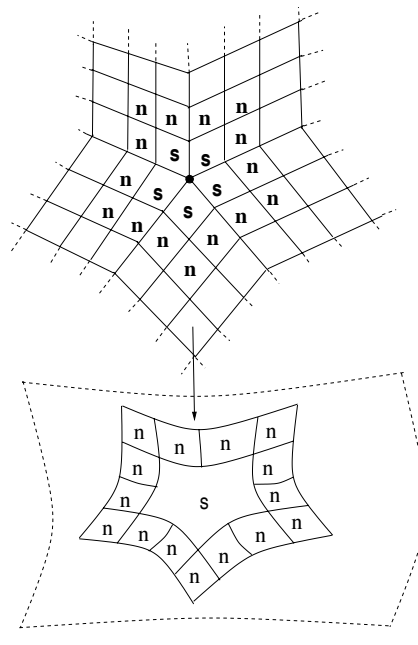

(a)

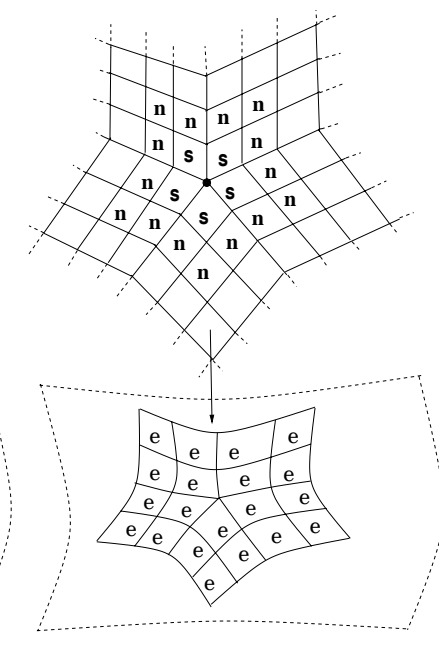

(b)
Figure 7: A control mesh with an extraordinary vertex of degree 5 and the corresponding limit surface: (a) using the concepts developed in $[14,19]$, where the limit surface consists of quadrilateral normal elements and a pentagonal special element; (b) using the unified approach developed in this paper, where the limit surface consists of one single type of quadrilateral finite element.

and $\mathbf{s}_{l}$ is the smooth quadrilateral patch corresponding to the $l$-th non-boundary quadrilateral face in the control mesh. Each of these quadrilateral patches can be parameterized over the corresponding non-boundary quadrilateral face in the control mesh. However, since a quadrilateral face can easily be reparameterized over a $[0,1]^{2}$ domain, each quadrilateral patch is locally parameterized over $[0,1]^{2}$.

The non-boundary quadrilateral faces are of two types : (a) faces having no extraordinary vertices (dubbed as "regular" faces in $[14,19]$, marked as $\kappa$ in Fig.8(a)) and (b) faces with one extraordinary vertex (dubbed as "irregular" faces in $[14,19]$, marked as $\zeta$ in Fig.8(a)). If there are $m$ regular and $n-m$ irregular faces, then Eqn.14 can be rewritten as

$$
\mathbf{s}=\sum_{i=1}^{m} \mathbf{s}_{i}+\sum_{j=1}^{n-m} \mathbf{s}_{j}
$$

where $\mathbf{s}_{i}$ is the quadrilateral patch corresponding to the $i$-th regular face and $\mathbf{s}_{j}$ is the quadrilateral patch corresponding to the $j$-th irregular face.

The quadrilateral patch in the limit surface corresponding to each regular face is a bicubic B-spline patch, which is defined over $[0,1]^{2}$. The set of control vertices defining this bicubic B-spline patch can be obtained using the adjacent face information. Therefore, the quadrilateral patches in the smooth limit surface corresponding to the regular faces in the control mesh can be easily expressed analytically, which are essentially bicubic B-spline patches defined by 16 control vertices over a $[0,1]^{2}$ domain. The analytic expression for the quadrilateral patch corresponding to the regular face $i$ is given by

$$
\begin{aligned}
\mathbf{s}_{i} & =\mathbf{J}_{b}(u, v) \mathbf{p}_{i} \\
& =\left(\mathbf{J}_{b}(u, v) \mathbf{A}_{i}\right) \mathbf{p} \\
& =\mathbf{J}_{i}(u, v) \mathbf{p}
\end{aligned}
$$




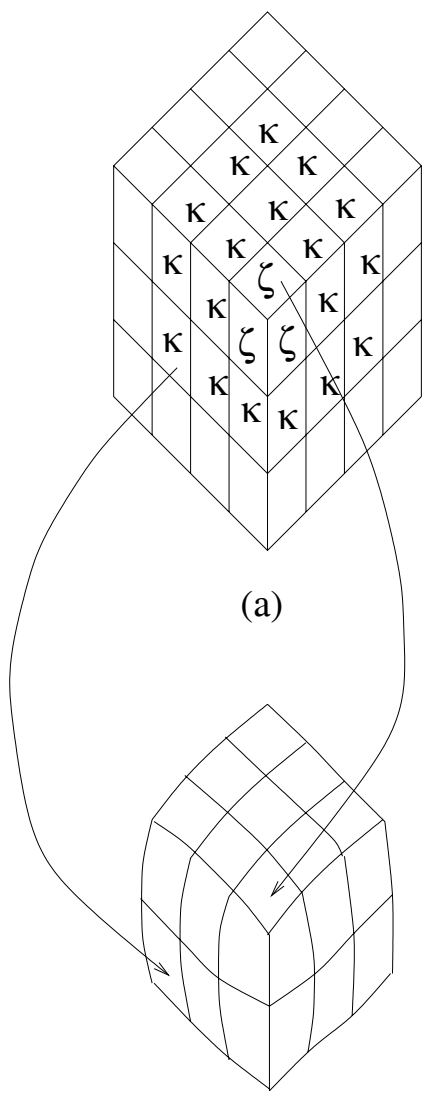

(b)

Figure 8: In Catmull-Clark subdivision, each non-boundary quadrilateral face in the control mesh has a corresponding quadrilateral patch in the limit surface : (a) control mesh, (b) limit surface.

where $0 \leq u, v \leq 1, \mathbf{J}_{b}(u, v)$ is the collection of the bicubic Bspline basis functions, $\mathbf{p}_{i}$ is the concatenation of the 16 control vertex positions defining the bicubic B-spline patch, $\mathbf{A}_{i}$ is a selection matrix which when multiplied with $\mathbf{p}$, the concatenation of all the control vertex positions defining the smooth limit surface, selects the corresponding set of control vertices, and $\mathbf{J}_{i}(u, v)=$ $\mathbf{J}_{b}(u, v) \mathbf{A}_{i}$.

By contrast, the analytic expression of the quadrilateral patches corresponding to the irregular faces in the control mesh was difficult to derive, and hence an alternative approach was taken in $[14,19]$. However, very recently an efficient scheme for evaluating CatmullClark subdivision surfaces at arbitrary parameter values has been proposed by Stam [23]. The proposed approach, involving eigenanalysis of the subdivision matrix, leads to an analytic expression of the quadrilateral patches which are parameterized over an irregular face in the control mesh, and hence over $[0,1]^{2}$ after reparameterization. Following the scheme developed by Stam [23], the quadrilateral patch corresponding to the irregular face $j$ is given by

$$
\begin{aligned}
\mathbf{s}_{j} & =\mathbf{J}_{d_{k}}(u, v) \mathbf{p}_{j} \\
& =\left(\mathbf{J}_{d_{k}}(u, v) \mathbf{A}_{j}\right) \mathbf{p} \\
& =\mathbf{J}_{j}(u, v) \mathbf{p},
\end{aligned}
$$

where $0 \leq u, v \leq 1$ as before. $\mathbf{J}_{d_{k}}(u, v)$ is the collection of basis functions for the corresponding quadrilateral patch in the smooth limit surface. The subscript $d_{k}$ is used to denote the fact that the irregular face has an extraordinary vertex of degree $k$. The de- tailed derivation and the analytic expressions of these basis functions involving the eigenvalues and eigenvectors of the subdivision matrix can be found in [23]. The other symbols used in Eqn.17 have the usual meaning: $\mathbf{p}_{j}$ is the concatenation of the $2 k+8$ control vertices defining the quadrilateral patch in the limit surface, $\mathbf{p}$ is the concatenation of all the control vertex positions defining the smooth limit surface, $\mathbf{A}_{j}$ is a selection matrix which when multiplied with $\mathbf{p}$ selects the corresponding set of control vertices, and $\mathbf{J}_{j}(u, v)=\mathbf{J}_{d_{k}}(u, v) \mathbf{A}_{j}$.

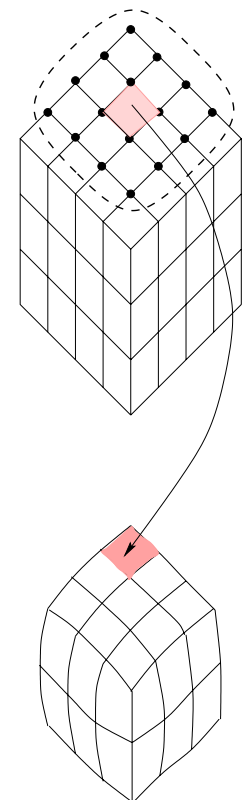

(a)

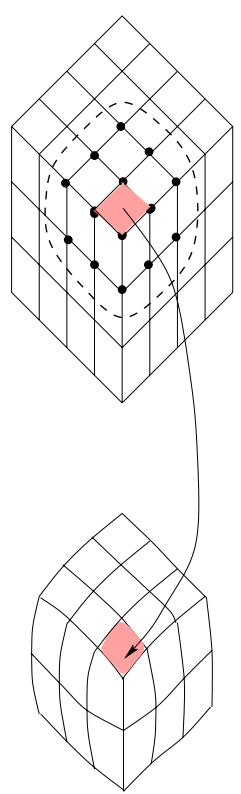

(b)
Figure 9: (a) The marked 16 control vertices define the shaded quadrilateral patch associated with the shaded regular face in the control mesh. (b) The marked 14 control vertices define the shaded quadrilateral patch associated with the shaded irregular face in the control mesh.

It may be noted that the number of control vertices in the initial mesh defining a quadrilateral patch in the smooth limit surface is $2 k+8$, where $k=4$ in case the associated quadrilateral face in the control mesh is regular, or $k=$ degree of the extraordinary vertex if the associated quadrilateral face is irregular. For example, the shaded quadrilateral patch is associated with the shaded regular face in Fig.2(a), and the 16 control vertices defining this patch (which is actually a bicubic B-spline patch) are marked. Similarly, the shaded quadrilateral patch is associated with the shaded irregular face in Fig.2(b), and the 14 control vertices defining this patch are highlighted. Now an expression of the smooth limit surface can be formulated. Using Eqn.15, 16 and 17, it can be shown that

$$
\begin{aligned}
\mathbf{s} & =\sum_{i=1}^{m} \mathbf{J}_{i} \mathbf{p}+\sum_{j=1}^{n-m} \mathbf{J}_{j} \mathbf{p} \\
& =\left(\sum_{i=1}^{m} \mathbf{J}_{i}+\sum_{j=1}^{n-m} \mathbf{J}_{j}\right) \mathbf{p} \\
& =\mathbf{J} \mathbf{p},
\end{aligned}
$$

where $\mathbf{J}=\left(\sum_{i=1}^{m} \mathbf{J}_{i}+\sum_{j=1}^{n-m} \mathbf{J}_{j}\right)$. Note that even though the initial mesh serves as the parametric domain of the smooth limit surface, each quadrilateral face in the initial mesh and consequently the smooth limit surface can be defined over a $[0,1]^{2}$ domain. 
Once an analytic expression of the smooth limit surface of Catmull-Clark subdivision is derived, we then develop the dynamic model by considering the control vertex positions as time-varying variables. The velocity of the surface model can be expressed as $\dot{\mathbf{s}}(\mathbf{x}, u, v)=\mathbf{J}(\mathbf{x}, u, v) \dot{\mathbf{p}}$, where an overstruck dot denotes a time derivative and $\mathrm{x} \in S^{0}, S^{0}$ being the domain defined by the initial mesh.

\subsection{Finite Element Implementation}

The smooth limit surface of Catmull-Clark subdivision comprises a collection of quadrilateral patches. Each quadrilateral patch is considered as a finite element. Therefore, within the unified framework the limit surface can be decomposed into one single type of finite elements rather than two different types as in $[14,19]$. Our new FEM technique significantly simplifies the data structure and system architecture. Consequently, more efficient algorithms for finite-element assembly, dynamic simulation, etc. can be devised using this unified approach. The motion equation of the dynamic model is same as that of the dynamic model of butterfly-based subdivision:

$$
\mathbf{M} \ddot{\mathbf{p}}+\mathbf{D} \dot{\mathbf{p}}+\mathbf{K} \mathbf{p}=\mathbf{f}_{p},
$$

where $\mathbf{f}_{p}$ is the generalized force vector and $\mathbf{M}, \mathbf{D}$, and $\mathbf{K}$ are the mass, damping and stiffness matrices of the model. The expressions of the mass, damping and stiffness matrices for a quadrilateral element (which is a bicubic B-spline) can be written as

$$
\begin{aligned}
& \mathbf{M}_{e}=\int_{0}^{1} \int_{0}^{1} \mu \mathbf{J}_{b}^{T} \mathbf{J}_{b} d u d v, \\
& \mathbf{D}_{e}=\int_{0}^{1} \int_{0}^{1} \gamma \mathbf{J}_{b}^{T} \mathbf{J}_{b} d u d v,
\end{aligned}
$$

and

$$
\begin{aligned}
\mathbf{K}_{e}= & \int_{0}^{1} \int_{0}^{1}\left(\alpha_{11}\left\{\left(\mathbf{J}_{b}\right)_{u}\right\}^{T}\left\{\left(\mathbf{J}_{b}\right)_{u}\right\}+\alpha_{22}\left\{\left(\mathbf{J}_{b}\right)_{v}\right\}^{T}\left\{\left(\mathbf{J}_{b}\right)_{v}\right\}\right. \\
& +\beta_{11}\left\{\left(\mathbf{J}_{b}\right)_{u u}\right\}^{T}\left\{\left(\mathbf{J}_{b}\right)_{u u}\right\}+\beta_{12}\left\{\left(\mathbf{J}_{b}\right)_{u v}\right\}^{T}\left\{\left(\mathbf{J}_{b}\right)_{u v}\right\} \\
& \left.+\beta_{22}\left\{\left(\mathbf{J}_{b}\right)_{v v}\right\}^{T}\left\{\left(\mathbf{J}_{b}\right)_{v v}\right\}\right) d u d v
\end{aligned}
$$

respectively, where $\mathbf{J}_{b}$ is the bicubic B-spline basis matrix, $\mu(u, v)$ is the mass density, $\gamma(u, v)$ is the damping density, $\alpha_{i i}(u, v)$ and $\beta_{i j}(u, v)$ are the tension and rigidity functions respectively. The subscript $u$ and $v$ denote partial derivatives with respect to $u$ and $v$ respectively. The subscript $e$ is used to indicate elemental matrices which are of size $(16,16)$. Note that, the mass, damping and stiffness matrices for these elements can be evaluated analytically, provided the material properties (e.g., mass, damping, rigidity and bending distributions) have analytic expressions. In some cases, these distribution functions can be assumed to be constant to simplify the matter.

The mass, damping and stiffness matrices for the quadrilateral elements which are not bicubic B-splines (corresponding to the irregular faces) can also be expressed analytically by simply replacing the matrix $\mathbf{J}_{b}$ in Eqn.20, 21 and 22 with the matrix $\mathbf{J}_{d_{k}}$ (refer to Eqn.17), where $k$ denotes the degree of the extraordinary vertex associated with the corresponding irregular face. These elemental matrices are of size $(2 k+8,2 k+8)$. The generalized force vector for these elements can also be determined in a similar fashion. It may be noted that the limits of integration need to be chosen carefully for elemental stiffness matrices as the second derivative diverges near the extraordinary points for Catmull-Clark subdivision surfaces.
Even though an analytical expression for a non-B-spline quadrilateral element in the limit surface exists, it is cumbersome to actually evaluate the elemental matrix expressions. Numerical integration using Gaussian quadrature may be used to obtain approximations of these elemental matrices. However, in this paper, an approach similar to the FEM procedure presented in Section 2 is utilized because of its simplicity and effectiveness. An approximation of the smooth limit surface is obtained by refining the initial control mesh $j$ times, and a spring-mass system is developed on this $j$-th approximation level in a similar fashion as in Section 2.3. The physical matrices of this system is then used as an approximation to the actual physical matrices. This approximation has been found to be very efficient for implementation purposes.

\section{Unified Approach For Any Subdivision Scheme}

The dynamic framework for modified butterfly and Catmull-Clark subdivision scheme can be generalized to any subdivision scheme. The key observation is that the smooth limit surface can be viewed as a collection of a single type finite element. Because of the nature of recursive refinement, any subdivision-based scheme essentially defines a "natural" correspondence which leads to a local parameterization of the smooth limit surface. The unique type of the associated finite element results from the local parameterization scheme. This is evident from the triangular finite element patches developed for the modified butterfly subdivision scheme and from the quadrilateral finite element patches developed for Catmull-Clark subdivision scheme. We shall present a general outline on how to provide a dynamic framework for interpolatory and approximating subdivision schemes.

\subsection{Interpolatory subdivision schemes}

Most of the interpolatory subdivision schemes are obtained by modifying the butterfly subdivision scheme [8]. Therefore, the framework for the modified butterfly subdivision scheme in Section 2 and its principles can be applied to other interpolatory subdivision schemes. The only difference is that the basis functions as well as the set of control vertices of arbitrary patch in the limit surface depend on the chosen interpolatory subdivision rules. It may also be noted that unlike the approximating schemes, the physical matrices can not be obtained analytically as the basis functions corresponding to interpolatory subdivision schemes do not have any analytic expressions in general. Even though these matrices can be obtained via numerical integration, the point-mass system connected by springs as developed in Section 2 is more preferable for implementation purposes because of efficiency reasons.

\subsection{Approximating subdivision schemes}

The unified approach for a dynamic model of Catmull-Clark subdivision can be generalized for other approximating subdivision schemes as well. This generalized approach involves three steps: (a) The limit surface obtained via an approximating subdivision scheme can be expressed as a collection of smooth patches which can be locally parameterized over a corresponding face in the control mesh. Each patch is $n$-sided if it is locally parameterized over a $n$-sided face. Analytic expressions for each of these patches can be derived even in the presence of extraordinary vertices in the control mesh, and hence an expression of the limit surface can be obtained. (b) Once an expression of the limit surface is obtained, the dynamic framework can be developed by considering control vertex positions as a function of time. The corresponding motion equation can be derived. 
(c) Each patch in the limit surface is treated as a finite element in implementation. The elemental mass, damping and stiffness matrices along with the generalized force vector can be obtained by either analytic or numerical integration. Alternatively, the control mesh can be subdivided $j$ times to obtain an approximation of the smooth limit surface, and a spring-mass system can be developed on this approximation mesh. The physical matrices of this system provide an approximation to the original physical matrices and works well in practice.

\section{Solid Modeling Applications}

The proposed FEM-based dynamic subdivision models can be used to represent a wide variety of smooth shapes with arbitrary genus. The smooth limit object can be sculpted by applying synthesized forces in a direct and intuitive way in shape design applications for solid modeling. The underlying shape from a cloud of 3D points can also be recovered hierarchically using our FEM models. For data fitting applications, springs are attached to the initialized model from the data points in $3 \mathrm{D}$, and the initialized model evolves dynamically according to the equation of motion subject to the applied spring forces and various geometric constraints. When an optimal fit to the given data set is achieved, the number of control vertices can be increased by replacing the original initial mesh by a new initial mesh obtained by applying a single subdivision step. This increases the number of degrees of freedom to represent the same limit surface and a new equilibrium position for the model with a much better fit to the given data set can be achieved. The fitting-error criteria for the discrete data can be computed according to distance between the data points and the points on the limit surface where the corresponding springs are attached. We now demonstrate modeling and data fitting examples using our dynamic FEM model.

In a shape modeling application, the user can specify any mesh as the initial (control) mesh, and the corresponding limit surface can be sculpted directly and interactively by applying synthesized forces in real-time. We show several initial surfaces obtained from different control meshes and the corresponding deformed surfaces after interactive sculpting on the limit surface in Fig.10 (see last page). To change the shape of an initial surface, the user can attach springs from different points in $3 \mathrm{D}$ to the nearest point on the limit surface such that the limit surface deforms towards these locations to generate the desired shape. The limit surface here consists of a single type of smooth triangular finite element patches, irrespective of the number of extraordinary vertices in the control mesh. The initial mesh of the smooth surface shown in Fig.10(a) has 125 faces and 76 vertices (degrees of freedom), which is deformed to the smooth shape shown in Fig.10(b) by interactive spring force application. The initial mesh of the closed solid shape in Fig.10(c) has 24 faces and 14 vertices. This solid shape is deformed to the shape shown in Fig.10(d). The one hole torus in Fig.10(e) and the corresponding modified shape in Fig.10(f) have initial meshes with 64 faces and 32 vertices. A two hole torus with a control mesh of 272 faces and 134 vertices, shown in Fig.10(g), is dynamically sculpted to the shape shown in Fig.10(h).

We have also performed several experiments testing the applicability of our model to recover the underlying shapes from a cloud of points in $3 \mathrm{D}$. In all the experiments, the initialized dynamic model has a control mesh comprising of 24 triangular faces and 14 vertices whereas the control mesh of the fitted model has 384 triangular faces and 194 vertices. It may be noted that once an optimal shape defined by a fixed number of control vertices (determined by subdivision levels) is recovered, the limit smooth model is capable of refining itself in accordance with the data-fitting criteria, thereby increasing the degrees of freedom of the recovered shape only when necessary. For the fitting-error (defined as the maximum distance between a data point and the nearest point on the limit surface expressed as a percentage of the diameter of the smallest sphere enclosing the object) of approximately $3 \%$, the initialized model is refined twice. The data-fitting examples are shown in Fig.11 (see last page). In the first data fitting experiment, range data acquired from multiple views of a light bulb is used and the model was initialized inside the 1000 data points (Fig.11(a)). The fitted dynamic model is shown in Fig.11(b). In the next experiment, the shape of a mechanical part is recovered from a range data-set containing 2031 data points (Fig.11(c) and (d)). We also recover the shape of a human head from the data set as shown in Fig.11(e). The head data set has 1779 3D points. It may be noted that the final shape with a very low error tolerance is recovered using very few number of control points in comparison to the large number of data points present in the original range data set.

\section{Conclusions}

In this paper, we have presented a new FEM-based dynamic framework where a single type of subdivision-based finite elements are used to represent the smooth limit surface generated by any subdivision scheme. The primary objective is to integrate physicsbased modeling techniques with geometric subdivision methodology for the interactive sculpting and direct manipulation of the limit surface of prevalent subdivision schemes. We have proposed an unified approach and demonstrated how to transform any subdivision scheme into our dynamic modeling framework. Modelers can physically sculpt virtual objects defined through arbitrary procedure-based subdivision techniques in a natural and intuitive manner within the proposed framework. Users can also directly enforce various functional and aesthetic requirements in the limit surface without the need to explicitly handle control vertices. Furthermore, this dynamic framework permits physics-based models to be refined adaptively in a hierarchical fashion which is an intrinsic feature of subdivision geometry. Our experiments have demonstrated the applicability of the new unified FEM-based framework in solid modeling and data fitting applications. This unified method will offer a greater potential for popular subdivision techniques in solid and geometric modeling, interactive graphics, finite element analysis, and engineering design applications.

\section{Acknowledgments}

This research was supported in part by the NSF grant ECS-9210648 and the NIH grant RO1-LM05944 to B.C. Vemuri; the NSF CAREER award CCR-9702103, the NSF grant DMI-9896170, and a research grant from Ford Motor Company to H. Qin. We wish to acknowledge Dr. Hughes Hoppe and Dr. Kari Pulli for the data sets.

\section{References}

[1] A.A. Ball and D.J.T. Storry, "Conditions for tangent plane continuity over recursively generated B-spline surfaces," ACM Transactions on Graphics, vol. 7, no. 2, pp. 83 - 102, 1988

[2] A.A. Ball and D.J.T. Storry, "An investigation of curvature variations over recursively generated B-spline surfaces," ACM Transactions on Graphics, vol. 9, no. 4, pp. 424 - 437, 1990.

[3] E. Catmull and J. Clark, "Recursively generated B-spline surfaces on arbitrary topological meshes," Computer Aided Design, vol. 10, no. 6, pp. $350-355,1978$. 
[4] G.M. Chaikin, "An algorithm for high speed curve generation," Computer Vision, Graphics and Image Processing, vol. 3, no. 4, pp. $346-349,1974$.

[5] T. DeRose, M. Kass and T. Truong, "Subdivision surfaces in character animation," in Computer Graphics Proceedings, ACM SIGGRAPH, Annual Conference Series, pp. 85 - 94, July, 1998.

[6] D. Doo, "A subdivision algorithm for smoothing down irregularly shaped polyhedrons," in Proceedings on Interactive Techniques in Computer Aided Design, pp. 157 - 165, 1978.

[7] D. Doo and M. Sabin, "Analysis of the behavior of recursive division surfaces near extraordinary points," Computer Aided Design, vol. 10, no. 6, pp. 356 - 360, 1978.

[8] N. Dyn, D. Levin and J.A. Gregory, "A butterfly subdivision scheme for surface interpolation with tension control," ACM Transactions on Graphics, vol. 9, no. 2, pp. 160 - 169, April, 1990.

[9] A. Habib and J. Warren, "Edge and vertex insertion for a class of $C^{1}$ subdivision surfaces," Computer Aided Geometric Design, to appear.

[10] M. Halstead, M. Kass and T. DeRose, "Efficient, fair interpolation using Catmull-Clark surfaces," in Computer Graphics Proceedings, ACM SIGGRAPH, Annual Conference Series, pp. 35 - 44, August, 1993.

[11] H. Hoppe, T. DeRose, T. Duchamp, M. Halstead, H. Jin, J. McDonald, J. Schweitzer and W. Stuetzle, "Piecewise smooth surface reconstruction," in Computer Graphics Proceedings, ACM SIGGRAPH, Annual Conference Series, pp. 295 - 302, July, 1994.

[12] C. Loop, Smooth subdivision surfaces based on triangles, M.S. thesis, University of Utah, Department of Mathematics, 1987.

[13] C. Mandal, A dynamic framework for subdivision surfaces, Ph.D. thesis, University of Florida, Gainesville, 1998.

[14] C. Mandal, H. Qin and B.C. Vemuri, "Dynamic smooth subdivision surfaces for data visualization," in IEEE Visualization'97 Conference Proceedings, Phoenix,AZ, pp. 371 - 377, October, 1997.

[15] C. Mandal, H. Qin and B.C. Vemuri. "Direct manipulation of butterfly subdivision surfaces : A physics-based approach," Technical Report CISE-TR-98-009, University of Florida, 1998.

[16] J. Peters and U. Reif, "The simplest subdivision scheme for smoothing polyhedra," ACM Transactions on Graphics, vol. 16, no. 4, pp. 420 - 431, October, 1997.

[17] J. Peters and U. Reif, "Analysis of generalized B-spline subdivision algorithms," SIAM Journal of Numerical Analysis, to appear, available at ftp://ftp.cs.purdue.edu/pub/jorg/9697agb.ps.Z.

[18] H. Qin and D. Terzopoulos, "D-NURBS : A physics-based framework for geometric design," IEEE Transactions on Visualization and Computer Graphics, vol. 2, no. 1, pp. 85 - 96, January - March, 1996.
[19] H. Qin, C. Mandal and B.C. Vemuri, "Dynamic CatmullClark subdivision surfaces," IEEE Transactions on Visualization and Computer Graphics, vol. 4, no. 3, pp. 215 - 229, July - September, 1998.

[20] U. Reif, "A unified approach to subdivision algorithms near extraordinary points," Computer Aided Geometric Design, vol. 12 , no. 2, pp. 153 - 174, 1995.

[21] J.E. Schweitzer, Analysis and Application of Subdivision Surfaces, Ph.D. thesis, University of Washington, Seattle, 1996.

[22] T.W. Sederberg, J. Zheng, D. Sewell and M. Sabin, "Nonuniform recursive subdivision surfaces," in Computer Graphics Proceedings, ACM SIGGRAPH, Annual Conference Series, pp. 387 - 394, July, 1998.

[23] J. Stam, "Exact evaluation of Catmull-Clark subdivision surfaces at arbitrary parameter values," in Computer Graphics Proceedings, ACM SIGGRAPH, Annual Conference Series, pp. 395 - 404, July, 1998.

[24] D. Terzopoulos, J. Platt, A. Barr and K. Fleischer, "Elastically deformable models," in Computer Graphics Proceedings, ACM SIGGRAPH, Annual Conference Series, pp. 205 $-214,1987$

[25] D. Zorin, P. Schröder and W. Sweldens, "Interpolating subdivision for meshes with arbitrary topology," in Computer Graphics Proceedings, ACM SIGGRAPH, Annual Conference Series, pp. 189 - 192, August, 1996.

[26] D. Zorin, "Smoothness of stationary subdivision on irregular meshes," Constructive Approximation, submitted, available as Stanford Computer Science Lab Tech. Rep. CSL-TR-98-752, 1998. 


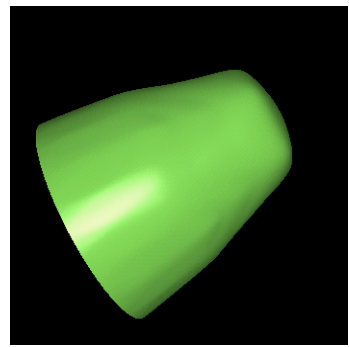

(a)

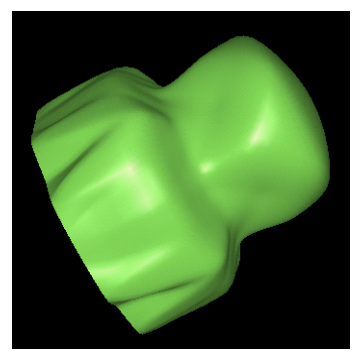

(b)

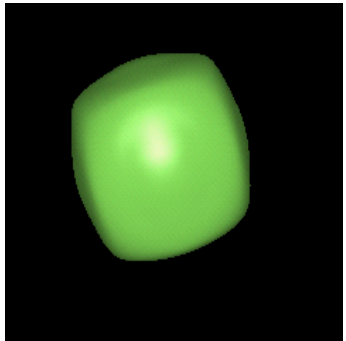

(c)

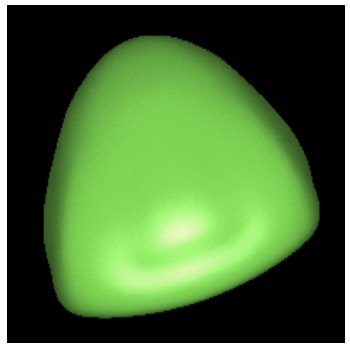

(d)

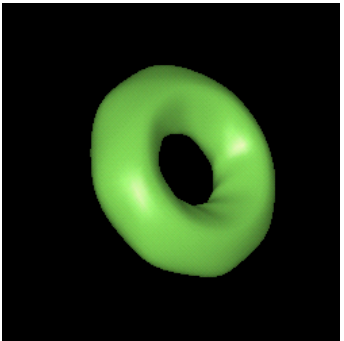

(e)

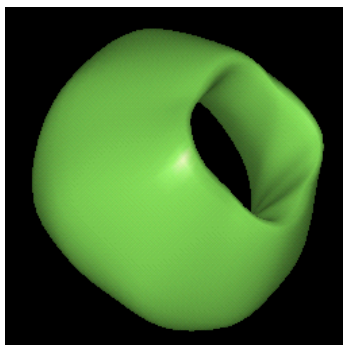

(f)

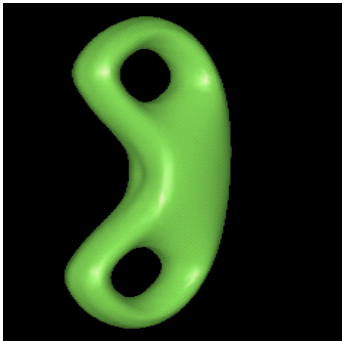

(g)

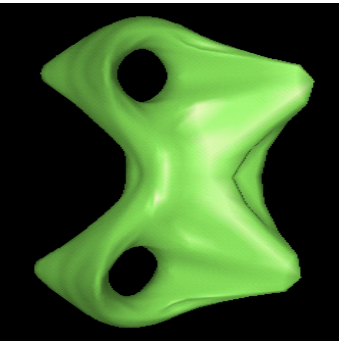

(h)

Figure 10: (a), (c), (e) and (g) : Initial shapes; (b), (d), (f) and (h) : the corresponding modified shapes after interactive sculpting via force application.

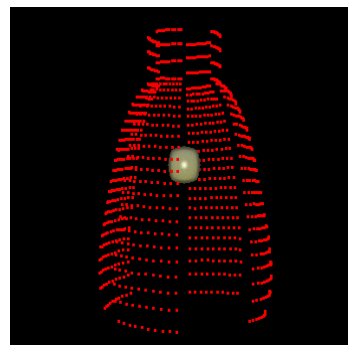

(a)

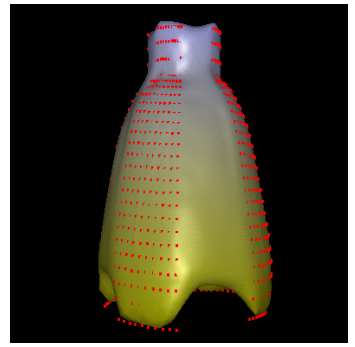

(b)

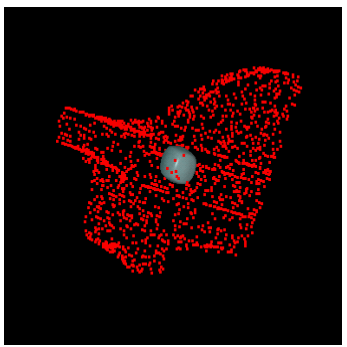

(c)

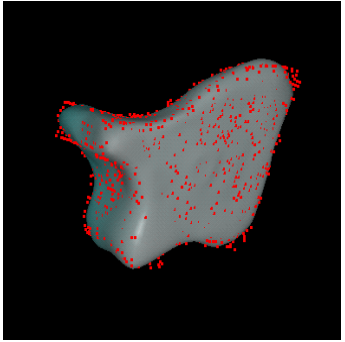

(d)

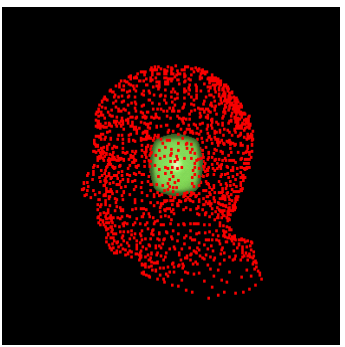

(e)

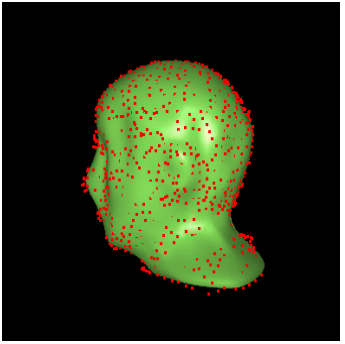

(f)

Figure 11: (a), (c) and (e) : Collection of points in 3D along with the initialized model; (b), (d) and (f) : the corresponding fitted dynamic subdivision surface model. 